\title{
FORTALECIMIENTO DEL SECTOR DE LA SOLDADURA EN LA PROVINCIA DE SANTA ELENA A TRAVES DE ACTIVIDADES DEL CENTRO DE CAPACITACION DE SOLDADURA LINDE-UPSE
}

\author{
Jorge Lucin Borbor ${ }^{(1)}$ \\ Carrera de Ingeniería Industrial. \\ ${ }^{(1)}$ Universidad Estatal Península de Santa Elena (UPSE) \\ Campus La Libertad, vía principal Santa Elena - La Libertad \\ La Libertad-Ecuador \\ jorge lucin@yahoo.com
}

\begin{abstract}
Resumen
La generación de grandes proyectos que tiene el país, en su ejecución lleva el aporte importante de la técnica de trabajo como es la soldadura. La Universidad Estatal Península de Santa Elena, UPSE y la multinacional LINDE, ex AGA S.A. Con el propósito de fortalecer el área de los trabajos de soldadura en la provincia de Santa Elena, firman un convenio de cooperación con una duración de 5 años. Para el efecto, se instala un Centro de Capacitación de primer orden con equipos, maquinarias, herramental e insumos necesarios para ofertar un trabajo de calidad. Los recursos físicos están dado por al UPSE, mientras que el conocimiento y técnica la da la multinacional por medio del facilitador- instructor de alta calificación. Los cursos a ofertar son de aprobación. El trabajo es organizado desde su difusión, inscripciones, capacitación, evaluación, aprobación e informes finales de cada curso, lo que se sustenta en las estadísticas y documentos soportes. Es así que, durante estos 3 primeros años de trabajo, se ha capacitado a 229 personas, con un total de 240 horas en 12 cursos de formación básica para soldadores de apoyo. Instituciones representativas en estos trabajos se han favorecido por la actualización de técnica y conocimientos impartidos en el Centro. Al convenio le quedan 1,5 años por terminar pero dado su fructífero trabajo, seguramente se renovara en pro de aportes a esta importante técnica de trabajo como la soldadura
\end{abstract}

Palabras claves: Convenio, capacitación, técnica, multinacional, aprobación

\begin{abstract}
The generation of grands projects that the country has, in its execution bears the important contribution of technical work such as welding. The University Santa Elena Peninsula, UPSE and multinational LINDE, ex AGA S.A., in order to strengthen the area of welding work in the province of Santa Elena, have signed a cooperation agreement with a duration of 5 years. For this purpose, is installed, an Training Center first order with equipment, machinery, tooling and supplies needed to offer quality work. Physical resources are given to UPSE, while knowledge and technique gives the multinational facilitator through highly qualified instructor. The courses to offering are approbation. The work is organized upon circulation, registration, training, evaluation, approval and final reports for each course, which is based on the statistics and media documents. Thus, over the first 3 years of work, has trained 229 people, with a total of 240 hours in 12 basic curs for welders support. Representative institutions in these works have been favored by updating technical knowledge and taught at the Center. The agreement remaining 1.5 years to finish but given its fruitful work, surely renew contributions towards this important technical work such as welding
\end{abstract}

Keywords: Convection, training, technical, multinational. Approval. 


\section{Introducción}

Los grandes proyectos que ejecuta el país en los últimos años, ven la necesidad de fortalecer un sinnúmero de actividades administrativas, técnicas, logísticas para poder cumplir con objetivos de aporte al desarrollo del país en busca del bienestar de la población. Algunos ejemplos están a la vista. La construcción de hidroeléctricas para la generación energía eléctrica económica y que ayude a la sustitución del consumo de gas por el uso de cocinas de inducción; construcción de vías, caminos y carreteras a lo largo del país para el movimiento oportuno de recursos; el proyecto Monteverde de almacenamiento en tierra de gas y su traslado hasta chorrillo para la minimizar de importaciones; el cambio de la matriz productiva para sustituir importaciones del sector productivo; la reforma e innovaciones a la educación secundaria y a nivel superior para el desarrollo del talento humano que busque la innovación y desarrollo del país; la construcción de la refinería del pacifico para la generación y diversificación de productos petroquímicos.

La actual provincia de Santa Elena, también es resultado de acciones que marcan el desarrollo de los pueblos. Desde su promulgación como tal, se ha visto claramente como el ámbito institucional se ha visto fortalecido en acciones y actividades que tienen influencia en el bienestar de la población. La Universidad Estatal Península de Santa Elena, creada en 1998, también ha sido participe y muchas veces líder de ese desarrollo con convenios y relaciones institucionales nacionales e internacionales. Dentro de sus unidades académicas, la Facultad de ingeniería industrial ha forjado el recurso humano técnico que aporta al país y dentro de sus convenios con instituciones, consideró oportuno que, colateralmente a la formación profesional debía fortalecerse el ámbito de un área técnica en las construcciones mecánicas y metalmecánicas de toda índole como son los trabajos de soldadura. Por ello, la idea de aportar con un centro de capacitación que actualiza y fortalezca el conocimiento y aplicaciones de esta técnica, toma forma. Es así que, en mayo del 2010, existen primeras conversaciones entre directivos de la UPSE y la internacional AGA S.A., las mismas que terminan en la firma de un convenio suscrito por el Ing. Jimmy Caldelll Soto en su calidad de rector de la UPSE y el Ing. Pablo Logroño, como Gerente de Operaciones de la multinacional. Posteriormente a ello, se designa al Ing. Jorge
Lucin Borbor como Coordinador del Centro de Capacitación de soldadura.

El Ing. Lucin, inicia la gestión para poder instalar un centro de capacitación con todos los recursos físicos para ofertar cursos. Se conversa con el Ing. Pablo Logroño de AGA S.A. y se definen necesidades de maquinarias, equipos e instalaciones para llevar adelante este proyecto En el mes de enero del 2011, la UPSE en coordinación con los técnicos de AGA S.A. se realiza las instalaciones de maquinarias, equipos, central de gases, las mismas que son probadas en el mes de febrero con la visita de técnicos.(Ing. Carlos Padilla e Ing. Wilmer Ramírez y directivos, Ing. Elio Rodríguez. Ing. Washington Vera, Telgo. Wartatanga) de ambas instituciones.

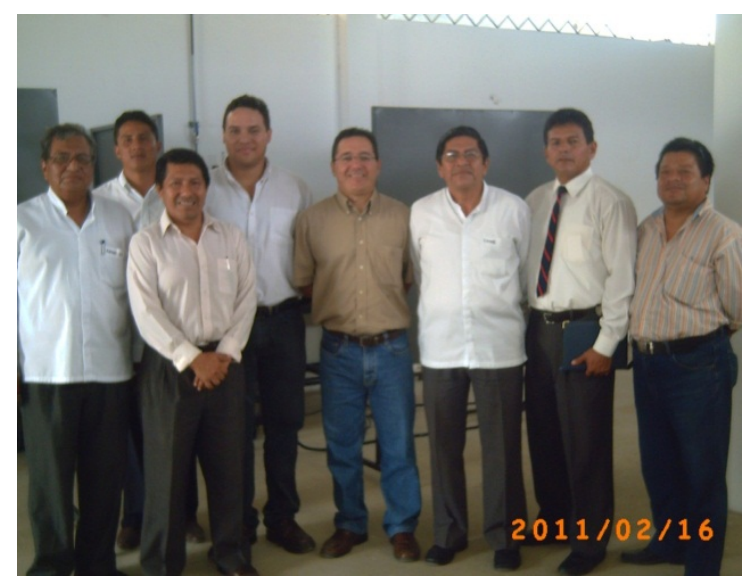

Fig. 1. Directivos de Linde S.A. y la UPSE

\section{Materiales y Métodos}

\subsection{Maquinarias, equipos y herramental del Centro de Capacitación}

La implementación de la maquinaria, equipo y herramental para el funcionamiento del Centro de capacitación, se la realizó en coordinación con técnicos del AGA S.A. y la UPSE. En primer lugar se adecuo un área construida de 6 metros por 12 metros y que servía como bodega secundaria. Allí, se instalaron 12 divisiones distribuidas así:

- 4 divisiones de dos por dos metros cada una para unidades de trabajo de oxiacetilénico y con mesa de trabajo

- 4 divisiones de dos metros por dos metros para unidades de trabajo con máquinas de soldar con mesa de trabajo

- 4 unidades de dos por dos metros para unidades de trabajo gases TIg y MIG con mesa de trabajo 
Internamente se distribuye ubicando en el centro una mesa general de trabajo y una cortadora mecánica de platinas.

En la parte posterior, se construye la central de gases para tanques de oxígeno, acetileno y que distribuyen por sistema de cañerías de 1 pulgadas a cada sitio de trabajo. En cada sito de trabajo, existen 4 terminales para la llegada de los gases cuando se trata de soldar oxiacetilénico y 8 terminales para soldar con argón

Se realizan las instalaciones eléctricas adecuadas y se adquieren insumos y equipos de protección personal si como también herramental y equipo menor de trabajo.

Las maquinas que se instalaron fueron

- 4 ESAB, Origo 328 AD/DC

- 4 Multimaster 300 AD/DC

\subsection{Promoción y Difusión}

En este mismo mes, comienza la promoción y difusión de los curso de soldadura a ofertar. Para ello, se considera el mercado laboral del área de soldadura que están las empresas que prestan servicios y talleres involucrados y los proyectos programados y en ejecución como el proyecto de almacenamiento de gas en Monteverde, la refinería del pacifico en Manta, los trabajos de mantenimiento de ductos y construcciones de tanques del sistema de Petroecuador en su filial Petro-Península. Se envían oficios a empresas como refinería la Libertad, Pacifpetrol, Proyecto de almacenamiento de gas Monteverde, Recubriteck S.A. Gremio de soldadores y dueños de talleres.

La difusión también consiste en mensajes radiales y en la página web de la universidad.

La afluencia de interesados se hizo presente

\subsection{Programa Académico}

De la conversación y coordinación entre las instituciones se establece el programa académico para las acciones de capacitación, asociados a los costó. Así tenemos que para la formación de un soldador de apoyo, es necesario que el aspirante tenga la siguiente secuencia académica que debe ser aprobada, mostrada en la tabla 1.
Tabla 1. Horas y costos de Curso Básico

\begin{tabular}{|l|l|c|}
\hline Nombre curso & Total de horas & Costo $\mathbf{( \$ )}$ \\
\hline $\begin{array}{l}\text { Soldadura y } \\
\text { corte }\end{array}$ & 4 teóricas & 55 \\
Oxiacetilénico & 16 practicas & \\
\hline $\begin{array}{l}\text { Soldadura por } \\
\text { arco } \\
\text { electrodos de } \\
\text { comunes }\end{array}$ & 16 teóricas & 55 \\
\hline $\begin{array}{l}\text { Soldadura por } \\
\text { arco de metal y } \\
\text { gas(MIG } \\
\text { MAG) }\end{array}$ & 16 teóricas & 70 \\
\hline $\begin{array}{l}\text { Soldadura por } \\
\text { arco } \\
\text { tungsteno y gas } \\
\text { TIG }\end{array}$ & 4 teóricas & 70 \\
\hline
\end{tabular}

La tabla muestra, que los cursos básicos, cada uno de ellos, tiene un total de 20 horas, de las cuales, 16 son sustento de práctica y ensayos y 4 son de sustento teórico. De tal forma que, un soldador básico debe completar 80 horas mínimas de capacitación. El cuadro refleja los costos iniciales de estos cursos que totalizan $\$ 250$.

A su vez, debe cumplir requisitos básicos como ser mayor de edad, copias de cedula y papel de votación actualizados y deseo de incursionar, aprender y actualizarse en esta técnica.

Además de ello, una vez cumplido la aprobación de este primer ciclo, el aspirante puede perfeccionar la técnica con otros cursos tales:

- como soladura blanda y fuerte;

- $\quad$ soldadura fuera de posición;

- soldadura de alambre tubular;

- soldadura de tubería con procesos diferentes como SMAW API 1104, SMAW ASME IX; SMAW AWS D1.1;

- Soldadura MIG(GMAW) con alambres especiales (inoxidable y aluminio

- Soldadura de tubería de aceros inoxidables con proceso

- Soldadura TIG (GTAW) con varillas especiales (inoxidable y aluminio.

De igual forma, se determina que de acuerdo a la experiencia llevada por AGA, S.A. en su escuela de soldadura en la ciudad de Guayaquil, se puede trabajar en procesos de calificación y para ello, el soldador una vez aprobado los cursos básicos y extras de formación, debe seguir otros cursos exclusivos para calificar a algún procedimiento técnico establecido como API, ASME o AWS. Entre estos cursos de servicios de calificación de 
soldadores de inspección de soldadura se establecen:

- $\quad$ Análisis de norma ASME ER IX y API 1104

- $\quad$ Con procesos de electrodos SMAW

- Con procesos MIG MAG.

- Con procesos TIG

- Con procesos de alambre tubular FCAW

- Con proceso BRAZING

- Con procesos mixtos.

Todos estos curso con una duración de 20 horas cada uno.

El procedimiento para aprobar un curso es que tenga un mínimo de 75 puntos y un máximo de 100 puntos. La ponderación en la calificación es 25 puntos en teoría y 75 puntos en la práctica Los primeros cuatro cursos son básicos y de allí el aspirante puede tomar los siguientes cursos para su perfeccionamiento. Solamente con la experiencia aplicativa laboral documentada, el aspirante puede estar en la capacidad de calificarse.

\subsection{Operatividad de los cursos.}

Para poder ejecutar un curso, y de acuerdo a la programación, se debe planificar algunas actividades. Cuando se define el programa fechas y horarios en coordinación con los instructores de AGA S.A., entonces se procede a una parte importante que es la promoción, difusión por medio de los radios y televisión local o nacionales, invitaciones a empresas e involucrados.

Cuando el interesado se acerca a buscar información, se incentiva a que escoja el curso en donde prácticamente va a aprender una nueva profesión o va a fortalecer los conocimientos. Decidido el interesado, se acerca al coordinador quien le extiende un documento-autorización para que pague el costo del curso en tesorería de la UPSE y luego regrese para inscribirlo, anexando copias de cedula y papel actualizado de votación.

El coordinador a su vez, solicitará con antelación a la dirección administrativa, la compra de los materiales para la práctica

Una vez inscritos los 20 participantes para el curso, este inicia. Generalmente el Centro de capacitación de Soldadura, CCS-UPSE-AGA, ha trabajado en horarios de $18 \mathrm{~h} 00$ a $22 \mathrm{~h} 00$, dado que todos los participantes de una u otra forma tiene sus actividades ocupadas en horarios diurno y vespertino.
El primer día de trabajo, trascurre en un aula y el facilitador, ing. Wilmer Ramirez, con listado en mano, hace la entrega de los folletos y trabaja con el sustento teórico de cada curso, detallando todo la técnica que se pondrá en práctica en los siguientes días. Así, ocurre los próximos días en que desde el inicio de las clase prácticas, en el taller adecuado para ello, se realizan probetas individuales y grupales bajo la atenta orientación del instructor, quien en la medida que avanza el trabajo, orienta, corrige, refuerza y evalúa los trabajos. El Centro provee de los materiales, insumos, equipos de protección personal, aperitivos que se utilizan en la práctica.

El día final de la capacitación, se realiza una evaluación escrita que complementa la calificación para que el aspirante apruebe el curso. Además de ello, el coordinador realiza una evaluación con respecto al trabajo del capacitador Posteriormente, el facilitador enviara su informe de la calificación final de los aspirantes y con ello, se elabora y de entrega un certificado de aprobación.

El coordinador emite los informes respectivos a las instancias involucradas, dejando documentado la vivencia de cada curso.

En las siguientes graficas se aprecia constancias de asistentes a la práctica de unos de los primeros cursos

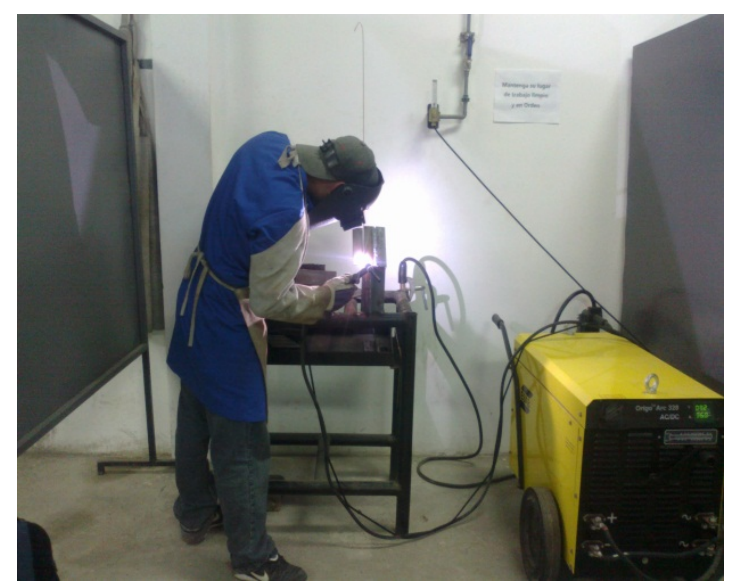




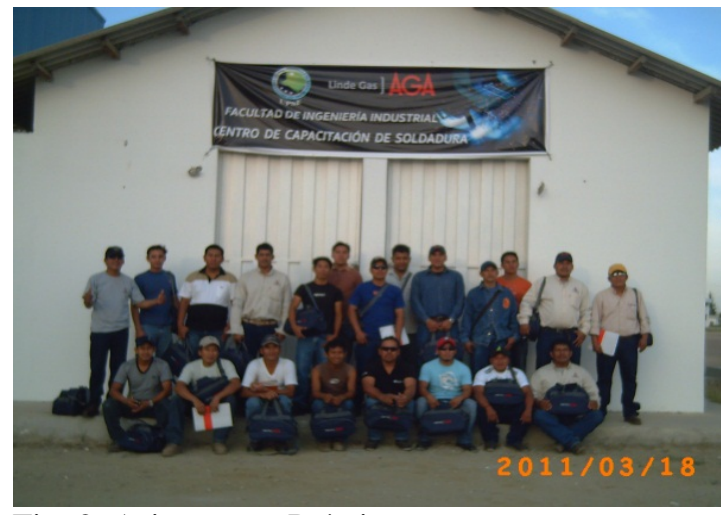

Fig. 2. Asistentes a Práctica

\section{Resultados}

\subsection{Estadística de los cursos ejecutados por el Centro de Capacitación de soldadura UPSE}

A continuación se presenta el cuadro estadístico de los cursos ejecutados

Total de horas trabajadas

En el siguiente cuadro, se expone los cursos y el total de horas trabajadas, desde marzo del 2011 a junio del 2013.
Tabla 2. Cursos y total de horas trabajadas

\begin{tabular}{|c|c|c|}
\hline FECHA & CURSO & $\begin{array}{c}\text { \# DE } \\
\text { HORA } \\
\text { S }\end{array}$ \\
\hline $\begin{array}{c}14 \text { al } 18 \\
\text { marzo/2011 }\end{array}$ & $\begin{array}{c}\text { soldadura } \\
\text { oxiacetilénica }\end{array}$ & 20 \\
\hline 4 al 8 abril/2011 & Soladura electrodos & 20 \\
\hline $\begin{array}{c}20 \text { al } 24 \\
\text { junio/2011 }\end{array}$ & $\begin{array}{r}\text { soldadura } \\
\text { MIGMAG }\end{array}$ & 20 \\
\hline $\begin{array}{c}4 \text { al } 8 \text { de } \\
\text { julio/2011 }\end{array}$ & $\begin{array}{c}\text { soldadura } \\
\text { oxiacetilénica }\end{array}$ & 20 \\
\hline $\begin{array}{c}8 \text { al } 11 \text { de } \\
\text { agosto/2011 }\end{array}$ & $\begin{array}{c}\text { soldadura } \\
\text { oxiacetilénica }\end{array}$ & 20 \\
\hline $\begin{array}{l}29 \text { agos-2 } \\
\text { Sep./2011 } \\
\end{array}$ & $\begin{array}{l}\text { soldadura } \\
\text { electrodos }\end{array}$ & 20 \\
\hline 10 al 14 oct $/ 11$ & soldadura TIG & 20 \\
\hline 21 al 25 nov/11 & $\begin{array}{c}\text { soldadura MIG } \\
\text { MAG }\end{array}$ & 20 \\
\hline $\begin{array}{c}23 \text { al } \\
\text { 27julio/2012 }\end{array}$ & $\begin{array}{c}\text { soldadura } \\
\text { oxiacetilénica }\end{array}$ & 20 \\
\hline $\begin{array}{l}5 \text { al } 9 \text { de } \\
\text { nov/2012 }\end{array}$ & $\begin{array}{c}\text { soldadura de } \\
\text { electrodos }\end{array}$ & 20 \\
\hline $\begin{array}{c}18 \text { al22 } \\
\text { marzo/2013 }\end{array}$ & $\begin{array}{c}\text { soldadura } \\
\text { oxiacetilénica }\end{array}$ & 20 \\
\hline 3 al 7 de junio/13 & $\begin{array}{l}\text { soldadura } \\
\text { electrodos }\end{array}$ & 20 \\
\hline TOTAL & & 240 \\
\hline
\end{tabular}

Fuente:

Estadística: CCS

La tabla 2, detalla los cursos ejecutados y el total de horas trabajadas. Desde el año 2011 al 2013, se han trabajado 12 cursos, de los cuales, 5 cursos son de soldadura oxiacetilénica para aceros al carbono; 4 de soldadura de electrodos comunes para aceros al carbono; 2 cursos de arco de gases MIG MAG y 1 curso de soldadura de arco de gastungsteno, TIG. El total de número de horas trabajadas 240 horas porque cada curso tiene una duración de 20 horas

\subsection{Número de participantes}

En el siguiente cuadro 3 se detalla el total de participantes inscritos que aprobaron o reprobaron los cursos 
Tabla 3. Participantes inscritos, aprobados y reprobados

\begin{tabular}{|c|c|c|c|c|}
\hline FECHA & CURSO & $\begin{array}{c}\text { total } \\
\text { partic. }\end{array}$ & $\begin{array}{l}\text { Partic } \\
\text { aprobados }\end{array}$ & $\begin{array}{l}\text { partic } \\
\text { reprobba }\end{array}$ \\
\hline $\begin{array}{c}14 \text { al } 18 \\
\text { marzo/2011 }\end{array}$ & $\begin{array}{c}\text { soldadura } \\
\text { oxiacetilénica }\end{array}$ & 24 & 22 & 2 \\
\hline $\begin{array}{c}4 \text { al } 8 \\
\text { abril//2011 }\end{array}$ & $\begin{array}{l}\text { Soldadura } \\
\text { electrodos }\end{array}$ & 22 & 22 & \\
\hline $\begin{array}{l}20 \text { al } 24 \\
\text { junio/2011 }\end{array}$ & $\begin{array}{l}\text { soldadura } \\
\text { MIGMAG }\end{array}$ & 15 & 15 & \\
\hline $\begin{array}{c}4 \text { al } 8 \text { de } \\
\text { julio/2011 }\end{array}$ & $\begin{array}{c}\text { soldadura } \\
\text { oxiacetilénica }\end{array}$ & 20 & 20 & \\
\hline $\begin{array}{c}8 \mathrm{al} 11 \mathrm{de} \\
\text { agosto/2011 }\end{array}$ & $\begin{array}{c}\text { soldadura } \\
\text { oxiacetilénica }\end{array}$ & 20 & 20 & \\
\hline $\begin{array}{c}29 \text { agos-2 } \\
\text { sep/2011 }\end{array}$ & $\begin{array}{l}\text { soldadura } \\
\text { electrodos }\end{array}$ & 20 & 19 & 2 \\
\hline $\begin{array}{c}10 \text { al } 14 \\
\text { oct } / 11\end{array}$ & soldadura TIG & 21 & 17 & 3 \\
\hline $\begin{array}{c}21 \text { al } 25 \\
\text { nov/11 }\end{array}$ & $\begin{array}{c}\text { soldadura Mig } \\
\text { Mag }\end{array}$ & 15 & 15 & \\
\hline $\begin{array}{c}23 \mathrm{al} \\
\text { 27julio/2012 }\end{array}$ & $\begin{array}{c}\text { soldadura } \\
\text { oxiacetilénica }\end{array}$ & 16 & 16 & \\
\hline $\begin{array}{c}5 \text { al } 9 \text { de } \\
\text { nov/2012 }\end{array}$ & $\begin{array}{c}\text { soldadura de } \\
\text { electrodos }\end{array}$ & 17 & 17 & \\
\hline $\begin{array}{c}18 \text { al22 } \\
\text { marz/2013 }\end{array}$ & $\begin{array}{c}\text { soldadura } \\
\text { oxiacetilénica }\end{array}$ & 21 & 21 & \\
\hline $\begin{array}{l}3 \text { al } 7 \text { de } \\
\text { junio/13 }\end{array}$ & $\begin{array}{l}\text { soldadura } \\
\text { electrodos }\end{array}$ & 18 & 17 & 1 \\
\hline TOTAL & & 229 & 221 & 8 \\
\hline
\end{tabular}

3.3. Relación de los cursos ofertados y de las condiciones de trabajo de los participantes

De las estadísticas y anotaciones llevadas por la coordinación, es importante resaltar el hecho de que de los 229 inscritos apenas el 3,06 \% de participantes, o sea 7 , se inscribieron como nuevos en adquirir el conocimiento y de ellos solo 2 siguieron los 4 cursos básicos. Los otros 5 no pudieron terminar los módulos de TIG y de MIG MAG, por el costo que representaba estos dos cursos finales.

Es decir, el 96,94 \% de participantes, o sea, 222 participantes tenían una relación de trabajo directa con el área de soldadura. De la indagación en el momento de la inscripción, ellos acotan que llevan años en el trabajo empírico y que su formación como una fuente de trabajo es per medio de su día a día en estos menesteres. Dicen que los cursos sirven para actualizar los conocimientos en máquinas modernas como tiene el Centro de Capacitación y que durante el desarrollo del curso han aprendido los importantes aspectos y secretos técnicos que tiene la soldadura. También acotan que durante sus trabajos y en la prestación de servicios, existen inconveniente con respecto a Fuente: certificados que solicitan sus demandantes y que consideran importantes que la actualización de sus conocimientos, se de por medio de un proceso de capacitación bien llevado por parte del Ing. Wilmer Ramírez, Técnico acreditado de AGA. S.

La tabla, muestra el total de participantes que se han inscritos en los curso y que han aprobado y reprobado. El total de participantes inscritos son de 229 y de ellos 221 han aprobados los cursos y apenas 8 ha reprobados, lo que representa un 3,49 $\%$. Es de recalcar que esta reprobación en todos los casos obedece a que el participante se ha retirado en el transcurso del curso y entonces no reúne el puntaje mínimo de aprobación que es de 75 puntos.

El cuadro también muestra que han participado de los cursos de soldadura oxiacetilénica un total de 101 participantes y de ellos solamente 2 no aprobaron. En tanto que, en los cursos de soldadura de electrodos comunes para aceros al carbono, han inscritos 77 personas y de ellos ha reprobado 3.

De los cursos de arco de MIG MAG, se han inscritos 30 participantes habiendo aprobados todos. Por ultimo en los curso de soldadura TIG, participaron 21 personas habiendo reprobado 3 .
A. y de la aprobación respaldado por una documentación de certificación firmado por directivos de AGA S.A. y de la UPSE.

\section{Conclusiones y Recomendaciones}

\section{Conclusiones:}

A finales del 2013, la multinacional AGA S.A. se convierte en LINDE., reflejando mayor grado de acción y representación a nivel mundial de esta multinacional alemana. El Centro de Capacitación de soldadura de la UPSE, nace como una necesidad de aportar en conocimientos y técnica actualizada a un sector importantes de trabajo técnico como es la soldadura. El convenio, suscrito en el 2010, tiene una duración de 5 años con grandes posibilidades de renovación dado el fructífero trabajo realizado acá en la provincia de Santa Elena. En la actualidad, el CCS UPSE LINDE, sigue trabajando en pos de seguir cumpliendo su objetivo y en el mes de junio del 
2014, se efectuó el primer curso de soldadura oxiacetilénica para aceros al carbono con una aceptación de 18 participantes que lo aprobaron. Se debe seguir trabajando y cumpliendo los objetivos. De los 229 inscritos, y con 211 aprobados de 229, nos demuestra que en estos dos años, este trabajo ha tenido una aceptación favorable y que las expectativas por seguir un proceso de capacitación en un área técnica importante e interesante están latentes para fortalecer una mano de obra necesaria en trabajos y proyectos de desarrollo.

\section{Recomendaciones}

Es pertinente que en Centro de Capacitación, siga:

1. Una aprobación como Centro apto para llevar adelante, procesos de Calificación para estos ámbitos de trabajos

2. Calificación de Docentes como instructores técnicos en el ámbito de soldadura.

3. Ejecute la aprobación de trabajos y proyectos en soldadura de alto nivel.

4. La calificación de personal apto para trabajos de alta calidad.

Siendo así, se prevé que siga funcionando bajo un esquema de mejorar el servicio técnico y con ello contribuir a fortalecer el ámbito técnico de la soldadura en la región y país

\section{Bibliografía.}

1. Nievel Benjamín, Freivalds Andris. 2010. Ingeniería Industrial. Métodos estándares y diseño de trabajo. McGraaw Hill.

2. López Rodríguez Ricardo 2011. Mecánica. Ediciones Tabor.

3. Gary Dessler. Administración de Recursos Humanos.2009. Pearson Prentice Hall

4. Creus Antonio (2011). Instrumentación Industrial.

5. Carpintero Pino José María. 2013. Soldadura Básica. Guía Práctica. Starbook

6. Álvarez Heredia Francisco; Faizal Geageas Enriqueta. 2012. Riesgos Laborales. Como prevenirlos en el ámbito de trabajo. Ediciones de la U.

7. Heizr Render. 2005. Principio de Administración de Operaciones. Pearson Prentice Hall.

8. Pacheco Chavira Jesús Neri. 2010. Mediciones

y Control de Procesos Industriales. Ediciones Trillas.
9. UPSE. 2010-2014. Documentos y estadísticas del trabajo del Centro de Capacitación de Soldadura. UPSE AGA.

10. AGA.S.A.2011. Normas técnicas de soldadura. Manual AGA S.A.

11. LINDE. 2013. Catálogos de soldadura y gases. 12. R.C. Hibbeler. Mecánica de materiales. 2010. Pearson. 